\title{
Aligned-gap multiple split ring resonator for dielectric sensing application
}

\begin{abstract}
This paper presents the design and development of planar Aligned-Gap Multiple Split Ring Resonator (SRR) for microwave sensor that operates at resonance frequency around $5 \mathrm{GHz}$. The sensor consists of a microstrip transmission line loaded with two elements of AlignedGap Multiple Split Ring Resonator (SRR) on both sides. The proposed sensor was designed and fabricated on Rogers RT5880 substrate having dielectric constant of 2.2 and thickness of $0.254 \mathrm{~mm}$. The final dimension of the proposed sensor was measured at $30 \times 15 \mathrm{~mm} 2$. Measured result show good agreement with simulated ones as well as exhibiting high Qfactor for use in sensing application. An obvious shift of resonance frequency is observed upon introduction of several samples with different dielectric value.
\end{abstract}

